\title{
ESTIMATION OF HETEROSIS AND COMBINING ABILITY FOR YIELD AND FIBER QUALITY TRAITS BY USING LINE X TESTER ANALYSIS IN COTTON (Gossypium barbadense L.)
}

\author{
Heba H.E. Hamed and S.R.N. Said \\ Cotton Research Institute, Agricultural Research Center, Giza, Egypt
}

Received: Dec. 31, 2020

Accepted: Jan. 27,2021

\begin{abstract}
The present study was carried out at Seds Agricultural Research Station, Cotton Research Institute, Agricultural Research Center, Egypt, during 2019 and 2020 seasons. This investigation was carried out to estimate heterosis, combining ability, proportional contributions, genetic components and heritability estimates of some characters for six Egyptian cotton varieties as lines i.e, Giza 80, Giza 86, Giza 90, Giza 93, Giza 94 and Giza 95, while, the other three genotypes used as testers were Karshenky, Ustraly 13 and Pima $S_{4}$, using line $x$ tester analysis. In 2020 season a randomized complete block design with three replications was carried to evaluate all genotype (nine parents and their $18 F_{1} s$ crosses) for some genetic parameters. The results indicated that mean squares due to the genotypes, parents, parents vs. crosses, crosses, lines, testers and Line $x$ Tester were highly significant for all studied traits, except boll weight, seed index and lint index at tester and fiber strength for Line $x$ Tester. The following crosses demonstrated the best heterosis relative to mid- and better-parent, i.e, Giza $80 \times$ Karashenky, Giza $86 \times$ Ustraly 13 and Giza $86 \times$ Pima $_{4}$ for most yield studied traits and the crosses Giza $93 \times$ Karashenky and Giza 93 x Ustraly 13 for most fiber quality traits. The results revealed that the lines Giza 86 and Giza 94 were significant and positive desirable GCA effects for most yield traits. Giza 93 had significant desirable GCA effects for all fiber traits, in this respect, the results of testers showed that Pima $S_{4}$ had significant desirable for some yield and fiber traits. However, estimates of specific combining ability (SCA) effects for crosses Giza 86 x Ustraly 13, Giza $90 \times$ Pima $\mathrm{S}_{4}$, Giza $93 \times$ Karshenky and Giza $95 \times$ Pima $S_{4}$ were significant desirable SCA effects for most yield traits, while, the crosses Giza $90 \times$ Pima $_{4}$, Giza 93 x Karshenky and Giza $95 \times$ Pima $\mathrm{S}_{4}$ were significant desirable SCA effects for most fiber traits. The results showed that proportion contribution of lines was higher than of lines $x$ tester interaction contribution and testers for all studied traits. The non-additive of genetic parameters was larger than additive genetic variance with respect to all studied traits except lint percentage, seed index, lint index and upper half mean. The highest broad sense heritability estimates was observed in case of UHM with values of $\mathbf{8 8 . 4 7 \%}$ and the lowest was for fiber strength with value of $32.24 \%$, while for narrow sense heritability, it was ranged from $8.04 \%$ to $49.03 \%$ for boll weight and upper half mean, respectively. Generally, Giza 86 and Giza 94 could be used in breeding programs for improving high yielding varieties, while Giza 93 could be considered as excellent parent for breeding programs to produce new varieties characterized with best fiber properties.
\end{abstract}

Key words: Cotton, Combining ability, Heterosis, Heritability, Gene action.

\section{INTRODUCTION}

Selection of superior parents with good combining ability for most of the yield contributing and quality parameters is the prime objective of any crop improvement programmes. Hence, identification of parents based on their combining ability is an important step to proceed further for hybridization and 
selection of superior segregates or to identify good hybrids for commercial exploitation. Line $x$ Tester design of crossing the genotypes is one of the tools which facilitates the plant breeder to identify superior genotypes and promising recombinants produced through estimation of General Combining Ability (GCA) and Specific Combining Ability (SCA). To choose appropriate parents and hybrids based on their combining ability estimates, the Line $x$ Tester method has been widely used by plant breeders in both self and cross pollinated crops Konak et al., (1999), Mert et al., (2003), Basbag et al., (2007), Ahuja and Dhayal (2007) and Basal et al., (2009). Sprague and Tatum (1942) used the term GCA to designate the average performance of a genotype in hybrid combinations and used the term SCA to define those cases in which, combinations do relatively better or worse than the expected on the basis of average performance of the genotypes involved.

In combining ability, the genetic variability of each trait can be partitioned into GCA and SCA Sprague and Tatum (1942). GCA effects explains about the additive type of gene action, whereas, SCA effects estimates the non additive (Dominant or epistasis) gene action. Importance of non additive gene action is observed for different yield contributing traits. However, appreciable degree of variance due to GCA was observed for morphological and yield traits Khan (2010). Many cotton cultivars despite their high/low agronomic performance combine in a better way/poorly when used as a parental cultivars in cross combinations Batool et al., (2010). Mabrouk et al., (2018) results revealed that the variances of the genotypes, parents and crosses were significant for bolls/plant, seed and lint cotton yield/plant, lint $\%$ and uniformity index characters. The mean squares due to GCA were significant for bolls/plant, seed and lint cotton yield/plant and lint \%, as well as mean squares of SCA were significant for all previous traits except lint \%. Recently, Balcha et al., (2019) estimate of variance analysis and showed that, presence of significant differences among genotypes for all studied traits except uniformity index, GCA (lines) was significant for all traits, while SCA was significant for number of bolls/plant, seed and lint cotton yield and fiber strength. Performing lines for lint yield and related traits followed by crossing with testers is possible to obtain commercial cotton hybrids. Also, Yehia and EL-Hashash (2019) reported that genotypes, parents $(P)$, crosses(C) and ( $P$ vs. $C)$ variances exhibited significantly differences $(P<0.01)$ for most studied characters. The variances due to GCA of parents, and SCA crosses were significant for most traits under study, indicating the importance of both additive and non-additive gene actions in controlling these traits. Line $\times$ Tester proportional contribution was greater than individual contribution of both lines and testers for most traits studied. ALHibbiny et al., (2020) cleared that highly significant and positive (desirable) heterosis relative to mid- and betterparents for most traits studied was found in the crosses Giza $89 \times 10229$ and Giza $96 \times$ 10229. On the other hand, the heterosis relative to mid- and betterparent was highly significant and negative (useful) for micronaire reading of the same crosses. High heritability in broad-sense estimates (>50\%) were detected for all the traits studied at the two crosses except seed cotton yield/plant at cross (Giza $89 \times 10229$ ) and boll weight of cross (Giza $96 \times 10229$ ). The heritability in narrow-sense estimates ranged from $3.29 \%$ to $35.70 \%$ for boll weight and uniformity index of cross (Giza 96 x 10229), respectively. 
The main objective of this study was to evaluate heterosis, combining ability, gene action and heritability for yield, yield components and fiber properties using Line $x$ Tester analysis in cotton (Gossypium barbadense L.).

\section{MATERIALS AND METHODS}

In 2019 growing season the single crosses between nine parental genotypes were made by using the six Egyptian cotton varieties, Giza 80, Giza 86, Giza 90, Giza 93, Giza 94 and Giza 95 as lines (Females). While, the three remaining varieties were used as testers (males) namely Karshenky (Russian variety), Ustraly 13 (Australian variety), and Pima $\mathrm{S}_{4}$ (American Egyptian variety) to produce $18 F_{1}$ 's and the parental varieties were also selfed to increase their seeds. Eighteen crosses and nine parents were evaluated in 2020 growing season at Seds Agricultural Research Station in an experiment randomized complete block design with three replications to evaluate genotypes. Each block therefore, contained 24 plots. Each plot was two rows $4 \mathrm{~m}$ long and $0.60 \mathrm{~m}$ wide. Hills were spaced $0.40 \mathrm{~m}$ apart which thinned to keep constant stand of one plant/hill.

\section{The studied traits were.}

Number of bolls per plant (NB/P), Seed cotton yield per plant (SCY/P.g), Lint cotton yield per plant (LCY/P.g), Lint percentage (L\%), Boll weight (BW.g), Seed index (SI g), Lint index (LI.g), Upper half mean (UHM), Micronaire reading (MIC), Fiber strength (FS) and Uniformity index (UI)

All fiber properties were measured in the laboratories of the Cotton Technology Research Division, Cotton Research Institute.

\section{Statistical analysis:}

The first step in the line $x$ tester analysis is to perform analysis of variance and test the significance of differences among the genotypes including crosses and parents. If these differences are found significant, line $x$ tester analysis was performed (Singh and Chaudhary 1979 and Kempthorne (1957), reported that, using broad base genotypes as a tester; the general combining of lines is tested as in the top cross method. They added that the line $x$ tester analysis is an extension of this method in which several testers are used. In order to evaluate the materials used in this study, means and variance of genotypes for the studied traits were calculated. Statistical procedures used in this study were done according to Cochran and Cox (1957). The significance of means was determined using the least significant difference value (L.S.D) at $\mathbf{0 . 0 5}$ and 0.01 levels of significance, according to the equation, which outlined by Steel and Torrie (1985). Heritability was estimated in both broad $\left(h^{2}{ }_{b} \%\right)$ and narrow $\left(h^{2} \%\right)$ senses from two formulas given by Allard (1960) and Mather (1949).

\section{RESULTS AND DISCUSSION}

\section{Analysis of variance}

The analysis of variance and the mean squares of all studied traits for the nine parents and their $18 F_{1}$ 's crosses are presented in Table (1). The results showed that the mean squares due to the genotypes, parents, parents vs. crosses, crosses, lines, testers and Line $x$ Tester were highly significant for all studied traits, except boll weight, seed index and lint index at tester and fiber strength for Line x Tester. Samreen et al., (2008) found that the GCA variances due to lines and testers and SCA due to lines $x$ testers interaction were significant for all studied characters. However, the magnitude of GCA variance for lines (females) and testers (pollinators) were higher than the SCA variance indicating preponderance of additive genes in the 
expression of all traits. Baloch et al., (2014) cleared that mean squares due to general combining ability (GCA) of lines and testers and specific combining ability (SCA) of lines $x$ tester interactions were significant. The significance of GCA and SCA variances suggested that both additive and dominant genes were controlling the studied characters. Swetha et al., (2018) noticed that analysis revealed significant GCA and SCA mean squares for all the traits except 2.5 percent span length. However GCA variance showed significant mean squares for all the traits except boll weight and uniformity ratio, and SCA showed significant mean squares for all the traits except micronaire and fiber strength.

Table 1. Mean squares of line $x$ tester analysis for yield, yield components and fiber properties.

\begin{tabular}{|c|c|c|c|c|c|c|c|}
\hline sov & df & $\mathrm{NB} / \mathrm{P}$ & SCY/P & LCY/P & L\% & BW & SI \\
\hline Replications & 2 & 5.16 & 42.82 & 9.29 & 0.21 & 0.00 & 0.03 \\
\hline Genotypes & 26 & $103.77^{\star \star}$ & $1491.16^{\star *}$ & $240.19^{\star *}$ & $7.00^{\star *}$ & $0.08^{\star \star}$ & $1.95^{\star \star}$ \\
\hline Parents & 8 & $164.72^{\star *}$ & $2067.23^{\star *}$ & $316.97^{\star *}$ & $10.02^{* *}$ & $0.08^{\star *}$ & $2.63^{\star \star}$ \\
\hline P. vs. C & 1 & $499.09 * *$ & $9169.59^{\star \star}$ & $1634.46^{\star \star}$ & $4.12^{\star \star}$ & $0.40^{* *}$ & $5.23^{\star \star}$ \\
\hline Crosses & 17 & $51.84^{\star \star}$ & $768.40^{\star *}$ & $122.04^{\star \star}$ & $5.75^{\star *}$ & $0.07^{\star \star}$ & $1.44^{\star \star}$ \\
\hline Lines & 5 & $115.51^{\star *}$ & $2035.14^{\star \star}$ & $304.12^{\star \star}$ & $17.73^{* *}$ & $0.17^{\star \star}$ & $4.70^{\star \star}$ \\
\hline Tester & 2 & $44.42^{\star \star}$ & $358.23^{\star \star}$ & $78.37^{\star \star}$ & $0.99^{* \star}$ & 0.01 & 0.04 \\
\hline Line $x$ Tester & 10 & $21.49^{\star \star}$ & $217.07^{\star \star}$ & $39.73^{\star \star}$ & $0.72^{\star \star}$ & $0.03^{\star \star}$ & $0.10^{*}$ \\
\hline Error & 52 & 3.36 & 24.46 & 4.09 & 0.18 & 0.01 & 0.04 \\
\hline
\end{tabular}

Table 1. Cont.

\begin{tabular}{|c|c|c|c|c|c|c|}
\hline \multicolumn{1}{|c|}{ SOV } & df & LI & UHM & FS & MIC & UI \\
\hline Replications & 2 & 0.05 & 0.02 & 0.01 & 0.00 & 0.11 \\
\hline Genotypes & 26 & $1.33^{\star \star}$ & $5.94^{\star \star}$ & $0.37^{\star \star}$ & $0.35^{\star \star}$ & $6.10^{\star \star}$ \\
\hline Parents & 8 & $2.14^{\star \star}$ & $7.12^{\star \star}$ & $0.64^{\star \star}$ & $0.54^{\star \star}$ & $10.71^{\star \star}$ \\
\hline P. vs. C & 1 & $3.89^{\star \star}$ & $4.27^{\star \star}$ & $1.21^{\star \star}$ & $0.29^{\star \star}$ & $2.21^{\star \star}$ \\
\hline Crosses & 17 & $0.80^{\star \star}$ & $5.49^{\star \star}$ & $0.19^{\star \star}$ & $0.27^{\star \star}$ & $4.15^{\star \star}$ \\
\hline Lines & 5 & $2.53^{\star \star}$ & $17.66^{\star \star}$ & $0.53^{\star \star}$ & $0.60^{\star \star}$ & $9.64^{\star \star}$ \\
\hline Tester & 2 & 0.04 & $0.49^{\star \star}$ & $0.08^{\star}$ & $0.20^{\star \star}$ & $2.37^{\star \star}$ \\
\hline Line x Tester & 10 & $0.08^{\star \star}$ & $0.40^{\star \star}$ & 0.04 & $0.12^{\star \star}$ & $1.77^{\star \star}$ \\
\hline
\end{tabular}


Estimation of heterosis and combining ability for yield and fiber quality traits....

\begin{tabular}{|c|c|c|c|c|c|c|}
\hline Error & 52 & 0.03 & 0.04 & 0.02 & 0.01 & 0.24 \\
\hline
\end{tabular}

The mean performance of genotypes

Mean performances for parents (lines and testers) and crosses are presented in Table (2). The lines Giza 86 had the highest values for fiber strength. Giza 93 had the best means for No. of bolls/plant, upper half mean, micronaire reading and uniformity index, Giza 94 had the best means for all yield studied traits except No. of bolls/plant and lint percentage, Giza 95 had the best means for lint percentage, while for testers. Karashenky had the best values for No. of bolls/plant and uniformity index, Ustraly 13 recorded the highest values for seed cotton yield/plant, lint cotton yield/plant, lint percentage and fiber strength, the tester pima $s_{4}$ had the highest values for boll weight, seed index, lint index, uper half mean and micronaire reading. The results also showed that the best mean performances were found for Giza $86 \times$ Ustraly $\mathbf{1 3}$ for lint cotton yield/plant, Giza $90 \times$ Pima $_{4}$ for No. of bolls/plant, Giza $93 \times$ Karshenky for micronaire reading and uniformity index, Giza 93 x Ustraly 13 for upper half mean and fiber strength, Giza $94 \times$ Karshenky for seed index and lint index, Giza $94 \times$ Pima $_{4}$ for seed cotton yield/plant and boll weight, Giza $95 \times \mathrm{Pima}_{4}$ for lint percentage.

\section{Heterosis:}

The diversity of genetic distance and different of originated was the important source for variability which lead to create new recombinations differently about the parent consequently finding heterosis. Heterosis expressed as the percentage deviation of $F_{1}$ mean performance relative to both mid and better-parents. Heterosis refers to the superiority of the $F_{1}$ hybrid in one or more characters over its parents, and lead to superiority in adaptation. In general, positive heterosis is considered as desirable for all studied traits, except micronaire reading. The magnitude of heterosis for all studied traits over the mid-parents (MP) and better parent (BP) was presented in Tables (3) and (4), respectively. For No. of bolls/plant 16 out of 18 crosses studied showed highly significant positive heterosis relative to mid-parent which ranged from $7.26 \%$ for Giza $93 \times$ Pima $_{4}$ to $48.32 \%$ for Giza $90 \times$ Pima $S_{4}$, eight crosses showed desirable heterosis relative to better-parent which ranged from $8.15 \%$ for Giza $86 \times$ Karshenky to $28.13 \%$ for Giza $90 \times$ Pima $S_{4}$. For seed cotton yield/plant relative heterosis versus mid-parent, 16 crosses out of 18 $F_{1}$ crosses possessed highly significant positive heterosis which ranged from $10.84 \%$ for Giza $93 \times$ Pima $S_{4}$ to $51.69 \%$ for Giza $90 \times$ Pima $S_{4}$, while nine crosses showed significant and positive heterosis relative to better-parent which ranged from $8.90 \%$ for Giza $94 \times$ Pima $S_{4}$ to $29.37 \%$ for Giza $90 \times$ Pima $S_{4}$. For lint cotton yield/plant the results of heterosis versus mid-parent revealed that sixteen crosses out of $18 F_{1}$ crosses were highly significant and positive heterosis which ranged from $13.62 \%$ for Giza $93 \times$ Pima $_{4}$ to $52.25 \%$ for Giza $90 \times$ Pima S4, while nine crosses showed highly significant positive heterosis relative to betterparent which ranged from $8.22 \%$ for Giza $94 \times$ Pima $_{4}$ to $31.12 \%$ for Giza $90 \times$ Pima $S_{4}$. In this respect, for lint percentage, the results showed that six crosses out of 18 $F_{1}$ crosses relative heterosis versus midparent were highly significant and positive which ranged from $1.76 \%$ for Giza 80 x Ustraly 13 to $4.24 \%$ for Giza 86 $x$ Pima $S_{4}$, whereas, heterosis versus 
better-parent showed that three crosses out of $18 \quad F_{1}$ crosses were highly significant and positive which ranged from $1.70 \%$ for Giza $80 \times$ Pima $S_{4}$ to $2.50 \%$ for Giza 86 x Ustraly 13. Regarding to boll weight the results of heterosis versus mid-parent revealed that 13 crosses out of $18 F_{1}$ crosses exhibited significant and positive heterosis, which ranged from $4.07 \%$ for Giza $90 \times$ Karshenky to $13.84 \%$ for Giza $86 x$ Karshenky, whereas, heterosis relative to better-parent showed that 5 crosses out of $18 F_{1}$ crosses were significant and positive which ranged from $4.55 \%$ for Giza 90 x Ustraly 13 to $7.93 \%$ for Giza 93 $x$ Ustraly 13.

Table 2. The mean performances of six parental lines, three testers and $18 F_{1}$ hybrids for yield, yield components and fiber properties.

\begin{tabular}{|l|c|c|c|c|c|c|}
\hline \multicolumn{1}{|c|}{ Genotypes } & NB/P & SCY/P & LCY/P & L\% & BW & SI \\
\hline Lines : & & & & & & \\
\hline Giza 80 & 34.97 & 108.80 & 43.85 & 40.30 & 3.12 & 10.50 \\
\hline Giza 86 & 38.54 & 128.47 & 52.17 & 40.54 & 3.34 & 10.43 \\
\hline Giza 90 & 38.89 & 122.57 & 46.40 & 37.87 & 3.15 & 10.93 \\
\hline Giza 93 & 49.36 & 149.03 & 52.56 & 35.26 & 3.02 & 9.43 \\
\hline Giza 94 & 44.45 & 150.17 & 60.00 & 39.97 & 3.39 & 11.33 \\
\hline Giza 95 & 43.00 & 138.97 & 57.73 & 41.57 & 3.23 & 9.97 \\
\hline Testers : & & & & & & \\
\hline Karashenky & 29.61 & 85.73 & 33.69 & 39.30 & 2.90 & 8.50 \\
\hline Ustraly 13 & 29.50 & 89.23 & 35.11 & 39.34 & 3.03 & 8.87 \\
\hline Pima S4 & 28.31 & 86.50 & 33.53 & 38.76 & 3.06 & 9.73 \\
\hline \multicolumn{1}{|c|}{ LSD 0.05 } & 3.00 & 8.10 & 3.31 & 0.69 & 0.13 & 0.32 \\
\hline LSD 0.01 & 4.00 & 10.80 & 4.41 & 0.92 & 0.18 & 0.42 \\
\hline F hybrids & & & & & & \\
\hline Giza 80 x Karshenky & 39.06 & 127.70 & 51.90 & 40.64 & 3.27 & 10.33 \\
\hline Giza 80 x Ustraly 13 & 37.14 & 114.87 & 46.55 & 40.52 & 3.09 & 10.70 \\
\hline Giza 80 x Pima S4 & 43.33 & 137.93 & 56.53 & 40.99 & 3.18 & 10.33 \\
\hline Giza 86 x Karshenky & 41.68 & 147.93 & 59.28 & 40.07 & 3.55 & 10.37 \\
\hline Giza 86 x Ustraly 13 & 45.71 & 157.70 & 65.54 & 41.56 & 3.45 & 10.23 \\
\hline Giza 86 x Pima S4 & 45.30 & 152.93 & 63.21 & 41.33 & 3.38 & 10.43 \\
\hline Giza 90 x Karshenky & 43.09 & 135.73 & 52.65 & 38.79 & 3.15 & 11.20 \\
\hline Giza 90 x Ustraly 13 & 43.03 & 141.83 & 54.40 & 38.35 & 3.30 & 11.10 \\
\hline Giza 90 x Pima S4 & 49.83 & 158.57 & 60.84 & 38.37 & 3.18 & 11.40 \\
\hline Giza 93 x Karshenky & 47.34 & 146.73 & 54.95 & 37.46 & 3.10 & 10.00 \\
\hline Giza 93 x Ustraly 13 & 43.94 & 143.53 & 54.35 & 37.87 & 3.27 & 10.13 \\
\hline Giza 93 x Pima S4 & 41.65 & 130.53 & 48.90 & 37.46 & 3.13 & 9.77 \\
\hline Giza 94 x Karshenky & 45.48 & 156.97 & 61.89 & 39.44 & 3.45 & 11.70 \\
\hline Giza 94 x Ustraly 13 & 45.28 & 152.47 & 60.09 & 39.41 & 3.37 & 11.37 \\
\hline Giza 94 x Pima S4 & 46.01 & 163.53 & 64.93 & 39.70 & 3.56 & 11.30 \\
\hline & & & & & & \\
\hline
\end{tabular}


Estimation of heterosis and combining ability for yield and fiber quality traits....

\begin{tabular}{|c|c|c|c|c|c|c|}
\hline Giza 95 x Karshenky & 35.49 & 113.50 & 45.88 & 40.42 & 3.20 & 9.53 \\
\hline Giza 95 x Ustraly 13 & 34.01 & 113.70 & 45.79 & 40.27 & 3.34 & 9.70 \\
\hline Giza 95 x Pima S4 & 40.63 & 129.03 & 53.90 & 41.77 & 3.18 & 9.50 \\
\hline LSD 0.05 & 2.60 & 7.02 & 2.87 & 0.60 & 0.12 & 0.27 \\
\hline LSD 0.01 & 3.47 & 9.35 & 3.82 & 0.79 & 0.16 & 0.36 \\
\hline
\end{tabular}

Table 2. Cont.

\begin{tabular}{|c|c|c|c|c|c|}
\hline Genotypes & LI & UHM & FS & MIC & UI \\
\hline \multicolumn{6}{|l|}{ Lines: } \\
\hline Giza 80 & 7.09 & 31.47 & 10.13 & 4.40 & 83.30 \\
\hline Giza 86 & 7.12 & 34.50 & 10.50 & 4.47 & 84.93 \\
\hline Giza 90 & 6.66 & 30.20 & 9.33 & 4.20 & 81.60 \\
\hline Giza 93 & 5.14 & 34.53 & 10.33 & 3.60 & 87.30 \\
\hline Giza 94 & 7.55 & 33.47 & 10.17 & 4.53 & 87.00 \\
\hline Giza 95 & 7.09 & 30.67 & 9.30 & 4.63 & 83.53 \\
\hline \multicolumn{6}{|l|}{ Testers : } \\
\hline Karashenky & 5.51 & 32.13 & 10.10 & 3.77 & 86.17 \\
\hline Ustraly 13 & 5.75 & 32.67 & 10.57 & 3.90 & 85.87 \\
\hline Pima S $_{4}$ & 6.16 & 32.70 & 10.30 & 3.53 & 85.77 \\
\hline LSD & 0.27 & 0.31 & 0.24 & 0.13 & 0.80 \\
\hline LSD $\quad 0.01$ & 0.37 & 0.41 & 0.32 & 0.18 & 1.06 \\
\hline \multicolumn{6}{|l|}{$F_{1}$ hybrids } \\
\hline Giza 80 x Karshenky & 7.08 & 32.57 & 10.27 & 4.17 & 86.07 \\
\hline Giza 80 x Ustraly 13 & 7.29 & 32.43 & 10.30 & 3.97 & 85.03 \\
\hline Giza $80 \times$ Pima S $_{4}$ & 7.18 & 32.80 & 10.07 & 3.80 & 84.60 \\
\hline Giza 86 x Karshenky & 6.93 & 33.77 & 10.70 & 4.30 & 86.83 \\
\hline Giza 86 x Ustraly 13 & 7.28 & 33.67 & 10.57 & 3.93 & 85.30 \\
\hline Giza $86 \times$ Pima S $_{4}$ & 7.35 & 33.23 & 10.43 & 3.77 & 87.00 \\
\hline Giza 90 x Karshenky & 7.10 & 31.47 & 10.20 & 4.03 & 83.73 \\
\hline Giza 90 x Ustraly 13 & 6.91 & 31.77 & 10.03 & 4.27 & 83.57 \\
\hline Giza $90 \times$ Pima $S_{4}$ & 7.10 & 31.13 & 10.17 & 3.80 & 85.17 \\
\hline Giza 93 x Karshenky & 5.99 & 35.60 & 10.70 & 3.40 & 87.27 \\
\hline Giza 93 x Ustraly 13 & 6.18 & 35.70 & 10.87 & 3.57 & 86.13 \\
\hline Giza $93 \times$ Pima S $_{4}$ & 5.85 & 34.77 & 10.57 & 3.53 & 86.87 \\
\hline Giza 94 x Karshenky & 7.62 & 32.77 & 10.40 & 4.03 & 84.47 \\
\hline Giza 94 x Ustraly 13 & 7.39 & 33.60 & 10.13 & 4.23 & 85.40 \\
\hline Giza 94 x Pima S & 7.44 & 32.73 & 10.33 & 4.40 & 86.57 \\
\hline Giza 95 x Karshenky & 6.47 & 31.57 & 10.23 & 4.27 & 84.53 \\
\hline Giza 95 x Ustraly 13 & 6.54 & 31.67 & 10.03 & 4.40 & 84.60 \\
\hline
\end{tabular}


Heba H.E. Hamed and S.R.N. Said

\begin{tabular}{|cc|c|c|c|c|c|}
\hline \multicolumn{2}{|c|}{ Giza 95 x Pima S } & 6.82 & 32.20 & 10.13 & 3.90 & 84.10 \\
\hline LSD & 0.05 & 0.24 & 0.27 & 0.21 & 0.12 & 0.69 \\
\hline LSD & 0.01 & 0.32 & 0.36 & 0.28 & 0.15 & 0.92 \\
\hline
\end{tabular}

Table 3. Heterosis relative to mid-parent (MP) for yield, yield components and fiber properties.

\begin{tabular}{|c|c|c|c|c|c|c|}
\hline Crosses & $\mathrm{NB} / \mathrm{P}$ & SCY/P & LCY/P & L\% & BW & SI \\
\hline Giza 80 x Karshenky & $20.96^{\star \star}$ & $31.29^{* \star}$ & $33.87^{\star \star}$ & $2.12^{\star \star}$ & $8.70^{\star \star}$ & $8.77^{\star \star}$ \\
\hline Giza 80 x Ustraly 13 & $15.21^{\star *}$ & $16.01^{\star *}$ & $17.91^{\star \star}$ & $1.76^{\star}$ & 0.71 & $10.50^{\star \star}$ \\
\hline Giza 80 x Pima S $_{4}$ & $36.94^{\star \star}$ & $41.25^{\star \star}$ & $46.12^{\star \star}$ & $3.69^{\star \star}$ & 3.08 & 2.14 \\
\hline Giza 86 x Karshenky & $22.32^{\star \star}$ & $38.13^{\star \star}$ & $38.08^{\star \star}$ & 0.38 & $13.84^{\star \star}$ & $9.51^{\star \star}$ \\
\hline Giza 86 x Ustraly 13 & $34.38^{\star \star}$ & $44.88^{\star \star}$ & $50.18^{\star \star}$ & $4.05^{\star \star}$ & $8.43^{\star \star}$ & $6.04^{\star \star}$ \\
\hline Giza $86 \times$ Pima S $_{4}$ & $35.54^{\star \star}$ & $42.29^{\star \star}$ & $47.51^{\star \star}$ & $4.24^{\star \star}$ & $5.58^{\star \star}$ & $3.47^{*}$ \\
\hline Giza 90 x Karshenky & $25.84^{\star \star}$ & $30.32^{\star *}$ & $31.48^{\star *}$ & 0.53 & $4.07^{\star}$ & $15.27^{\star \star}$ \\
\hline Giza 90 x Ustraly 13 & $25.85^{\star \star}$ & $33.93^{\star \star}$ & $33.47^{\star \star}$ & -0.65 & $6.69 * \star$ & $12.12^{\star \star}$ \\
\hline Giza 90 x Pima S ${ }_{4}$ & $48.32^{\star \star}$ & $51.69^{\star \star}$ & $52.25^{\star \star}$ & 0.15 & 2.47 & $10.32^{\star \star}$ \\
\hline Giza 93 x Karshenky & $19.90^{\star \star}$ & $25.00^{\star \star}$ & $27.43^{\star \star}$ & 0.47 & $4.73^{*}$ & $11.52^{\star \star}$ \\
\hline Giza 93 x Ustraly 13 & $11.45^{\star \star}$ & $20.48^{\star \star}$ & $24.00^{\star \star}$ & 1.51 & $8.05^{\star \star}$ & $10.75^{\star \star}$ \\
\hline Giza $93 \times$ Pima S 4 & $7.26^{\star}$ & $10.84^{\star \star}$ & $13.62^{\star *}$ & 1.21 & 3.07 & 1.91 \\
\hline Giza 94 x Karshenky & $22.84^{\star \star}$ & $33.08^{\star \star}$ & $32.13^{\star \star}$ & -0.48 & $9.76^{\star \star}$ & $17.98^{\star \star}$ \\
\hline Giza 94 x Ustraly 13 & $22.46^{\star \star}$ & $27.37^{\star \star}$ & $26.37^{\star \star}$ & -0.62 & $5.09^{\star \star}$ & $12.54^{\star \star}$ \\
\hline Giza 94 x Pima S & $26.47^{\star \star}$ & $38.20^{\star \star}$ & $38.85^{\star \star}$ & 0.86 & $10.34^{\star \star}$ & $7.28^{\star \star}$ \\
\hline Giza 95 x Karshenky & -2.22 & 1.02 & 0.36 & -0.03 & $4.35^{*}$ & $3.25^{\star}$ \\
\hline Giza 95 x Ustraly 13 & -6.17 & -0.35 & -1.35 & -0.45 & $6.82^{\star \star}$ & $3.01^{*}$ \\
\hline Giza 95 x Pima S $_{4}$ & $13.96^{\star \star}$ & $14.46^{\star \star}$ & $18.12^{\star \star}$ & $4.01^{\star \star}$ & 0.95 & $-3.55^{\star}$ \\
\hline $\begin{array}{ll}\text { LSD } & 0.05\end{array}$ & 2.60 & 7.02 & 2.87 & 0.60 & 0.12 & 0.27 \\
\hline LSD & 3.47 & 9.35 & 3.82 & 0.79 & 0.16 & 0.36 \\
\hline
\end{tabular}

Table 3. Cont.

\begin{tabular}{|c|c|c|c|c|c|}
\hline Crosses & LI & UHM & FS & MIC & UI \\
\hline Giza 80 x Karshenky & $12.34^{\star \star}$ & $2.41^{\star \star}$ & 1.48 & 2.04 & $1.57^{\star \star}$ \\
\hline Giza 80 x Ustraly 13 & $13.54^{\star \star}$ & $1.14^{\star *}$ & -0.48 & $-4.42^{\star \star}$ & 0.53 \\
\hline Giza 80 x Pima S $_{4}$ & $8.34^{\star \star}$ & $2.23^{\star \star}$ & -1.47 & $-4.20^{\star \star}$ & 0.08 \\
\hline Giza 86 x Karshenky & $9.84^{\star \star}$ & $1.35^{\star \star}$ & $3.88^{* *}$ & $4.45^{\star \star}$ & $1.50^{* \star}$ \\
\hline Giza 86 x Ustraly 13 & $13.11^{\star \star}$ & 0.25 & 0.32 & $-5.98^{\star \star}$ & -0.12 \\
\hline Giza 86 x Pima S & $10.74^{\star \star}$ & -1.09 & 0.32 & $-5.83^{\star \star}$ & $1.93^{\star \star}$ \\
\hline Giza 90 x Karshenky & $16.62^{\star \star}$ & $0.96^{\star}$ & $4.97^{\star *}$ & 1.26 & -0.18 \\
\hline Giza 90 x Ustraly 13 & $11.25^{\star \star}$ & $1.06^{*}$ & 0.84 & $5.35^{\star \star}$ & -0.20 \\
\hline Giza $90 \times$ Pima S $_{4}$ & $10.71^{\star \star}$ & -1.01 & $3.57^{\star \star}$ & -1.72 & $1.77^{\star \star}$ \\
\hline Giza 93 x Karshenky & $12.50^{\star \star}$ & $6.80^{\star \star}$ & $4.73^{\star \star}$ & $-7.69^{\star \star}$ & 0.61 \\
\hline Giza 93 x Ustraly 13 & $13.42^{\star \star}$ & $6.25^{\star \star}$ & $3.99^{\star \star}$ & $-4.89^{\star \star}$ & -0.52 \\
\hline Giza $93 \times$ Pima $_{4}$ & 3.55 & $3.42^{\star \star}$ & $2.42^{\star}$ & -0.93 & 0.39 \\
\hline Giza 94 x Karshenky & $16.75^{\star \star}$ & -0.10 & $2.63^{\star}$ & $-2.81^{\star}$ & $-2.44^{\star \star}$ \\
\hline Giza 94 x Ustraly 13 & $11.19^{\star \star}$ & $1.61^{\star \star}$ & $-2.25^{\star}$ & 0.40 & $-1.20^{\star \star}$ \\
\hline Giza $94 \times$ Pima S & $8.56^{* *}$ & $-1.06^{\star}$ & 0.98 & $9.09^{* *}$ & 0.21 \\
\hline
\end{tabular}


Estimation of heterosis and combining ability for yield and fiber quality traits....

\begin{tabular}{|c|c|c|c|c|c|}
\hline Giza 95 x Karshenky & 2.68 & 0.53 & $5.50^{\star \star}$ & 1.59 & -0.37 \\
\hline Giza 95 x Ustraly 13 & 1.87 & 0.00 & 1.01 & $3.12^{\star}$ & -0.12 \\
\hline Giza 95 x Pima S & 2.88 & $1.63^{\star *}$ & $3.40^{\star *}$ & $-4.49^{\star *}$ & -0.65 \\
\hline LSD 0.05 & 0.24 & 0.27 & 0.21 & 0.12 & 0.69 \\
\hline LSD 0.01 & 0.32 & 0.36 & 0.28 & 0.15 & 0.92 \\
\hline
\end{tabular}

*,** Significant and highly significant at 0.05 and 0.01 probability levels, respectively.

Table 4. Heterosis relative to better-parents (BP) for yield, yield components and fiber properties.

\begin{tabular}{|c|c|c|c|c|c|c|}
\hline Crosses & NB/P & SCY/P & LCY/P & $L \%$ & BW & SI \\
\hline Giza 80 x Karshenky & $11.67^{\star \star}$ & $17.37^{\star \star}$ & $18.36^{\star \star}$ & 0.85 & $4.92^{*}$ & -1.59 \\
\hline Giza 80 x Ustraly 13 & 6.19 & 5.58 & 6.16 & 0.55 & -0.75 & 1.90 \\
\hline Giza 80 x Pima S & $23.88^{\star \star}$ & $26.78^{\star \star}$ & $28.92^{\star \star}$ & $1.70^{*}$ & 2.14 & -1.59 \\
\hline Giza 86 x Karshenky & $8.15^{*}$ & $15.15^{\star \star}$ & $13.62^{\star \star}$ & -1.16 & $6.39^{\star \star}$ & -0.64 \\
\hline Giza 86 x Ustraly 13 & $18.62^{\star \star}$ & $22.76^{\star \star}$ & $25.62^{\star \star}$ & $2.50^{\star \star}$ & 3.40 & -1.92 \\
\hline Giza 86 x Pima S & $17.55^{\star \star}$ & $19.05^{\star \star}$ & $21.16^{\star \star}$ & $1.95^{\star}$ & 1.20 & 0.00 \\
\hline Giza 90 x Karshenky & $10.82^{\star \star}$ & $10.74^{\star \star}$ & $13.47^{\star \star}$ & -1.29 & -0.11 & 2.44 \\
\hline Giza 90 x Ustraly 13 & $10.66^{\star \star}$ & $15.72^{\star \star}$ & $17.23^{\star \star}$ & $-2.51^{\star *}$ & $4.55^{*}$ & 1.52 \\
\hline Giza $90 \times \mathrm{Pima} \mathrm{S}_{4}$ & $28.13^{\star \star}$ & $29.37^{\star \star}$ & $31.12^{\star \star}$ & -0.99 & 0.95 & $4.27^{\star *}$ \\
\hline Giza 93 x Karshenky & -4.09 & -1.54 & 4.56 & $-4.68^{\star \star}$ & 2.65 & $6.01^{\star \star}$ \\
\hline Giza 93 x Ustraly 13 & $-10.97^{\star \star}$ & -3.69 & 3.41 & $-3.75^{\star \star}$ & $7.93^{\star \star}$ & $7.42^{\star \star}$ \\
\hline Giza $93 \times$ Pima $_{4}$ & $-15.61^{\star *}$ & $-12.41^{\star *}$ & $-6.95^{\star}$ & $-3.35^{\star *}$ & 2.40 & 0.34 \\
\hline Giza 94 x Karshenky & 2.33 & 4.53 & 3.16 & -1.32 & 1.87 & $3.24^{*}$ \\
\hline Giza 94 x Ustraly 13 & 1.87 & 1.53 & 0.16 & -1.40 & -0.49 & 0.29 \\
\hline Giza 94 x Pima S & 3.51 & $8.90^{\star \star}$ & $8.22^{\star \star}$ & -0.67 & $5.02^{\star}$ & -0.29 \\
\hline Giza 95 x Karshenky & $-17.45^{\star \star}$ & $-18.33^{\star \star}$ & $-20.54^{\star \star}$ & $-2.77^{\star \star}$ & -1.03 & $-4.35^{\star \star}$ \\
\hline Giza 95 x Ustraly 13 & $-20.90^{\star *}$ & $-18.18^{\star \star}$ & $-20.68^{\star \star}$ & $-3.12^{\star \star}$ & 3.40 & -2.68 \\
\hline Giza $95 \times$ Pima S 4 & -5.51 & $-7.15^{\star}$ & $-6.64^{*}$ & 0.49 & -1.75 & $-4.68^{\star \star}$ \\
\hline $\begin{array}{ll}\text { LSD } & 0.05\end{array}$ & 3.00 & 8.10 & 3.31 & 0.69 & 0.13 & 0.32 \\
\hline 0.01 & 4.00 & 10.80 & 4.41 & 0.92 & 0.18 & 0.42 \\
\hline
\end{tabular}

Table 4. Cont.

\begin{tabular}{|c|c|c|c|c|c|}
\hline Crosses & LI & UHM & FS & MIC & UI \\
\hline Giza 80 x Karshenky & -0.20 & $1.35^{\star \star}$ & 1.32 & $10.62^{\star \star}$ & -0.12 \\
\hline Giza 80 x Ustraly 13 & 2.82 & -0.71 & $-2.52^{*}$ & 1.71 & $-0.97^{\star}$ \\
\hline Giza $80 \times$ Pima S $_{4}$ & 1.23 & 0.31 & -2.27 & $7.55^{\star *}$ & $-1.36^{\star \star}$ \\
\hline Giza 86 x Karshenky & -2.58 & $-2.13^{\star \star}$ & 1.90 & $14.16^{\star \star}$ & 0.77 \\
\hline Giza 86 x Ustraly 13 & 2.25 & $-2.42^{\star \star}$ & 0.00 & 0.85 & -0.66 \\
\hline Giza 86 x Pima S 4 & 3.29 & $-3.67^{\star \star}$ & -0.63 & $6.60^{\star \star}$ & $1.44^{\star \star}$ \\
\hline Giza 90 x Karshenky & $6.50^{\star *}$ & $-2.07^{\star \star}$ & 0.99 & $7.08^{\star *}$ & $-2.82^{\star \star}$ \\
\hline Giza 90 x Ustraly 13 & 3.62 & $-2.76^{* \star}$ & $-5.05^{\star *}$ & $9.40^{* *}$ & $-2.68^{\star \star}$ \\
\hline Giza 90 x Pima $S_{4}$ & $6.52^{\star \star}$ & $-4.79^{\star \star}$ & -1.29 & $7.55^{\star \star}$ & -0.70 \\
\hline Giza 93 x Karshenky & $8.74^{\star \star}$ & $3.09^{* \star}$ & $3.55^{\star \star}$ & $-5.56^{\star \star}$ & -0.04 \\
\hline Giza 93 x Ustraly 13 & $7.39^{\star *}$ & $3.38^{\star *}$ & $2.84^{\star}$ & -0.93 & $-1.34^{\star *}$ \\
\hline Giza 93 x Pima S 4 & -5.03 & 0.68 & $2.26^{*}$ & 0.00 & -0.50 \\
\hline Giza 94 x Karshenky & 0.97 & $-2.09^{\star *}$ & $2.30^{*}$ & $7.08^{\star \star}$ & $-2.91^{\star \star}$ \\
\hline Giza 94 x Ustraly 13 & -2.05 & 0.40 & $-4.10^{\star \star}$ & $8.55^{\star \star}$ & $-1.84^{\star \star}$ \\
\hline Giza 94 x Pima S ${ }_{4}$ & -1.43 & $-2.19^{\star \star}$ & 0.32 & $24.53^{\star \star}$ & -0.50 \\
\hline
\end{tabular}


Heba H.E. Hamed and S.R.N. Said

\begin{tabular}{|c|c|c|c|c|c|c|}
\hline \multicolumn{2}{|c|}{ Giza 95 x Karshenky } & $-8.78^{\star \star}$ & $-1.76^{\star \star}$ & 1.32 & $13.27^{\star \star}$ & $-1.90^{\star \star}$ \\
\hline \multicolumn{2}{|c|}{ Giza 95 x Ustraly 13} & $-7.76^{\star \star}$ & $-3.06^{\star \star}$ & $-5.05^{\star \star}$ & $12.82^{\star \star}$ & $-1.48^{\star \star}$ \\
\hline \multicolumn{2}{|c|}{ Giza 95 x Pima S } & -3.88 & $-1.53^{\star \star}$ & -1.62 & $10.38^{\star \star}$ & $-1.94^{\star \star}$ \\
\hline LSD & 0.05 & 0.27 & 0.31 & 0.24 & 0.13 & 0.80 \\
\hline LSD & 0.01 & 0.37 & 0.41 & 0.32 & 0.18 & 1.06 \\
\hline
\end{tabular}

*,** Significant and highly significant at 0.05 and 0.01 probability levels, respectively.

Concerning seed index, the results of heterosis versus mid-parent revealed that 15 of 18 crosses were exhibited significant positive heterosis which ranged from $3.01 \%$ for Giza $95 \times$ Ustraly 13 to $17.98 \%$ for Giza 94 x Karshenky, whereas, heterosis versus better-parent showed that four crosses were positive and significant which ranged from $3.24 \%$ for Giza 94 x Karshenky to $7.42 \%$ for Giza $93 \times$ Ustraly 13. For lint index the results of heterosis versus mid-parent revealed that 14 crosses out of $18 F_{1}$ crosses were found to be significant and positive heterosis which ranged from $8.34 \%$ for Giza $80 \times$ Pima $_{4}$ to $16.75 \%$ for Giza $94 \times$ Karshenky, but for heterosis versus better-parent showed that 4 out of 18 crosses were significant and positive which ranged from $6.50 \%$ for Giza $90 \times$ Karshenky to $8.74 \%$ for Giza $93 \times$ Karshenky. Regarding to upper half mean the results of heterosis versus midparent revealed that 11 crosses out of 18 $F_{1}$ crosses were found to be significant and positive heterosis which ranged from $1.06 \%$ for Giza 90 x Ustraly 13 to $6.80 \%$ for Giza 93 x Karshenky, whereas, heterosis versus better-parent showed that 3 of 18 crosses were exhibited highly significant positive heterosis which ranged from $1.35 \%$ for Giza $80 \times$ Karshenky to $3.38 \%$ for Giza 93 x Ustraly 13. Concerning fiber strength the results of heterosis versus mid-parent revealed that 9 of 18 crosses were exhibited significant positive heterosis which ranged from $2.42 \%$ for Giza $93 \times$ Pima $\mathrm{S}_{4}$ to $5.50 \%$ for Giza $95 \times$ Karshenky, whereas, heterosis versus better-parent showed that 4 of 18 crosses were exhibited significant positive heterosis which ranged from $2.26 \%$ for Giza $93 \times$ Pima $\mathrm{S}_{4}$ to $3.55 \%$ for Giza $93 \mathrm{x}$ Karshenky. Regarding to micronaire reading the results of heterosis versus mid-parent revealed that 8 of 18 crosses were exhibited significant negative heterosis which ranged from $-2.81 \%$ for Giza 94 x Karshenky to $-7.69 \%$ for Giza 93 $x$ Pima $S_{4}$, whereas, heterosis versus better-parent showed that the cross Giza $93 \times$ Karshenky was highly significant negative heterosis with value $-5.56 \%$. For uniformity index the results of heterosis versus mid-parent revealed that 4 crosses out of 18 crosses were exhibited significant positive heterosis which ranged from $1.50 \%$ for Giza $86 \mathrm{x}$ Karshenky to $1.93 \%$ for Giza 86 x Pima $\mathrm{S}_{4}$, whereas, heterosis versus betterparent showed that the cross Giza $86 \mathrm{x}$ Pima $\mathrm{S}_{4}$ was highly significant positive heterosis with value $1.44 \%$.

Lingaraja (2017) results showed that range of economic heterosis varied from 1.58 to $32.91 \%$ of seed index, 11.15 to $31.85 \%$ of lint index, -11.06 to $3.37 \%$ of ginning outturn, -6.32 to $8.80 \%$ of 2.5 per cent span length, $-\mathbf{2 . 7 3}$ to 18.27 of fiber strength, 17.69 to 21.23 of micronaire value, -2.08 to 1.66 of fiber uniformity and -60.38 to 48.32 of seed cotton yield per plant. AL-Ameer (2015) showed that the following crosses were evidenced the best values of heterosis relative to better and mid-parents i.e., crosses; TNB x Giza 85 and CB-58 x Giza 85 for most studied characters. Mahrous (2018) the results of heterosis noticed that 7 crosses had positive and highly significant heterosis in seed and lint cotton yield /plant and number of bolls/plant i.e., (Giza 80 x Giza 90), (G.86 x G.90), (G.86 x G.95), (G.87 x 
Estimation of heterosis and combining ability for yield and fiber quality traits....

G.90), (G.45 x (G.90 x Australian)), and (G. $92 \times$ G.90).

\section{Combining ability}

The estimates of general combining ability and specific combining ability are presented in Table (5) and Table (6), respectively. The results revealed that the line Giza 80 was significant desirable for lint percentage and lint index. Giza 86 was significant desirable for all studied traits except seed index and micronaire reading. Giza 90 was significant desirable for No. of bolls/plant, seed cotton yield/plant, seed index and lint index. Giza 93 had significant desirable GCA effects for No. of bolls/plant, upper half mean, fiber strength and uniformity index and negative desirable for micronaire reading. Giza 94 had significant and positive desirable GCA effects for all studied traits except lint percentage. Giza 95 had significant and positive desirable GCA effects for lint percentage. In this respect, the results of testers showed that Karshenky had significant and positive desirable GCA effects for fiber strength. Ustraly 13 had significant and positive desirable for upper half mean. Pima $\mathrm{S}_{4}$ showed significant desirable GCA effects for No. of bolls/plant, seed cotton yield/plant, lint cotton yield/plant, lint percentace, micronaire reading and uniformity index.

Table 5. Estimates of general combining ability effects of the parental genotypes for yield, yield components and fiber traits.

\begin{tabular}{|c|c|c|c|c|c|c|}
\hline Parents & NB/P & SCY/P & LCY/P & L \% & BW & SI \\
\hline Lines : & & & & & & \\
\hline Giza 80 & $-2.83^{\star \star}$ & $-13.46^{\star \star}$ & $-3.98^{\star \star}$ & $1.03^{\star \star}$ & $-0.10^{\star \star}$ & -0.05 \\
\hline Giza 86 & $1.57^{\star}$ & $12.57^{\star \star}$ & $7.03^{\star \star}$ & $1.30^{\star \star}$ & $0.17^{\star \star}$ & $-0.16^{\star}$ \\
\hline Giza 90 & $2.65^{\star \star}$ & $5.09^{\star \star}$ & 0.32 & $-1.19^{\star \star}$ & $-0.08^{\star \star}$ & $0.73^{\star \star}$ \\
\hline Giza 93 & $1.64^{\star \star}$ & -0.02 & $-2.91^{\star \star}$ & $-2.10^{\star \star}$ & $-0.12^{\star \star}$ & $-0.54^{\star \star}$ \\
\hline Giza 94 & $2.92^{\star \star}$ & $17.37^{\star \star}$ & $6.66^{\star \star}$ & -0.17 & $0.17^{\star \star}$ & $0.95^{\star \star}$ \\
\hline Giza 95 & $-5.96^{\star \star}$ & $-21.54^{\star \star}$ & $-7.12^{\star \star}$ & $1.13^{\star \star}$ & $-0.05^{\star}$ & $-0.93^{\star \star}$ \\
\hline LSD 0.05 & 1.23 & 3.31 & 1.35 & 0.28 & 0.05 & 0.13 \\
\hline LSD 0.01 & 1.63 & 4.41 & 1.80 & 0.37 & 0.07 & 0.17 \\
\hline Testers : & & & & & & \\
\hline Karashenky & -0.64 & -2.19 & $-1.22^{\star}$ & $-0.22^{\star}$ & 0.001 & 0.02 \\
\hline Ustraly 13 & $-1.15^{\star}$ & $-2.94^{\star}$ & $-1.19^{\star}$ & -0.03 & 0.02 & 0.03 \\
\hline Pima S & $1.79^{\star \star}$ & $5.13^{\star \star}$ & $2.41^{\star \star}$ & $0.25^{\star}$ & -0.02 & -0.05 \\
\hline LSD 0.05 & 0.87 & 2.34 & 0.96 & 0.20 & 0.04 & 0.09 \\
\hline LSD 0.01 & 1.16 & 3.12 & 1.27 & 0.26 & 0.05 & 0.12 \\
\hline
\end{tabular}

Table 5. Cont.

\begin{tabular}{|c|c|c|c|c|c|}
\hline Parents & LI & UHM & FS & MIC & UI \\
\hline \multicolumn{6}{|l|}{ Lines: } \\
\hline Giza 80 & $0.26^{\star \star}$ & $-0.37^{\star \star}$ & $-0.13^{\star \star}$ & -0.01 & -0.17 \\
\hline Giza 86 & $0.27^{\star *}$ & $0.59^{\star \star}$ & $0.23^{\star \star}$ & 0.01 & $0.98^{\star *}$ \\
\hline Giza 90 & $0.12^{\star}$ & $-1.51^{* *}$ & $-0.21^{\star *}$ & $0.05^{\star}$ & $-1.25^{\star \star}$ \\
\hline Giza 93 & $-0.91^{\star \star}$ & $2.39^{\star \star}$ & $0.37^{\star \star}$ & $-0.49^{\star \star}$ & $1.35^{\star \star}$ \\
\hline Giza 94 & $0.57^{\star \star}$ & 0.06 & -0.05 & $0.24^{\star \star}$ & 0.08 \\
\hline Giza 95 & $-0.31^{\star \star}$ & $-1.16^{\star \star}$ & $-0.21^{\star \star}$ & $0.20^{\star *}$ & $-0.99^{\star \star}$ \\
\hline LSD $\quad 0.05$ & 0.11 & 0.13 & 0.10 & 0.05 & 0.33 \\
\hline $\begin{array}{ll}\text { LSD } & 0.01\end{array}$ & 0.15 & 0.17 & 0.13 & 0.07 & 0.43 \\
\hline
\end{tabular}


Heba H.E. Hamed and S.R.N. Said

\begin{tabular}{|c|c|c|c|c|c|}
\hline \multicolumn{6}{|l|}{ Testers: } \\
\hline Karashenky & -0.05 & -0.01 & $0.08^{*}$ & $0.05^{\star \star}$ & 0.08 \\
\hline Ustraly 13 & 0.01 & $0.17^{\star \star}$ & -0.02 & $0.07^{\star \star}$ & $-0.40^{\star \star}$ \\
\hline Pima S & 0.04 & $-0.16^{\star \star}$ & -0.06 & $-0.12^{\star \star}$ & $0.31^{\star *}$ \\
\hline $\begin{array}{ll}\text { LSD } & 0.05\end{array}$ & 0.08 & 0.09 & 0.07 & 0.04 & 0.23 \\
\hline 0.01 & 0.11 & 0.12 & 0.09 & 0.05 & 0.31 \\
\hline
\end{tabular}

${ }^{\star, * \star}$ significant and highly significant at 0.05 and 0.01 probability levels, respectively.

Table 6. Estimates of specific combining ability effects of the $18 F_{1}$ crosses for yield, yield components and fiber traits.

\begin{tabular}{|c|c|c|c|c|c|c|}
\hline Crosses & NB/P & SCY/P & LCY/P & $L \%$ & BW & SI \\
\hline Giza 80 x Karshenky & -0.14 & 3.06 & 1.46 & 0.15 & 0.09 & -0.14 \\
\hline Giza 80 x Ustraly 13 & -1.55 & $-9.03^{\star \star}$ & $-3.92^{\star \star}$ & -0.17 & $-0.11^{*}$ & 0.21 \\
\hline Giza $80 \times$ Pima S & 1.70 & $5.97^{\star}$ & $2.46^{*}$ & 0.02 & 0.02 & -0.07 \\
\hline Giza 86 x Karshenky & -1.91 & -2.73 & -2.18 & $-0.70^{\star \star}$ & 0.09 & 0.01 \\
\hline Giza 86 x Ustraly 13 & $2.63^{*}$ & $7.78^{\star \star}$ & $4.05^{\star \star}$ & $0.60^{*}$ & -0.03 & -0.14 \\
\hline Giza $86 \times$ Pima S $_{4}$ & -0.72 & -5.06 & -1.88 & 0.10 & -0.06 & 0.14 \\
\hline Giza 90 x Karshenky & -1.58 & $-7.45^{\star}$ & -2.09 & $0.50^{*}$ & -0.06 & -0.05 \\
\hline Giza 90 x Ustraly 13 & -1.14 & -0.61 & -0.38 & -0.12 & 0.07 & -0.17 \\
\hline Giza $90 \times$ Pima $_{4}$ & $2.72^{\star}$ & $8.06^{\star *}$ & $2.47^{\star}$ & -0.38 & -0.01 & $0.22^{\star}$ \\
\hline Giza 93 x Karshenky & $3.67^{\star *}$ & $8.66^{* *}$ & $3.43^{* *}$ & 0.08 & -0.07 & 0.02 \\
\hline Giza 93 x Ustraly 13 & 0.78 & $6.21^{*}$ & $2.81^{\star}$ & 0.30 & 0.08 & 0.13 \\
\hline Giza 93 x Pima S $_{4}$ & $-4.45^{\star \star}$ & -14.87 & $-6.24^{\star \star}$ & -0.38 & -0.02 & -0.15 \\
\hline Giza 94 x Karshenky & 0.54 & 1.51 & 0.81 & 0.14 & -0.01 & $0.23^{*}$ \\
\hline Giza 94 x Ustraly 13 & 0.84 & -2.25 & -1.02 & -0.08 & $-0.11^{*}$ & -0.12 \\
\hline Giza 94 x Pima S & -1.37 & 0.74 & 0.21 & -0.06 & $0.12^{*}$ & -0.11 \\
\hline Giza 95 x Karshenky & -0.58 & -3.05 & -1.43 & -0.18 & -0.04 & -0.06 \\
\hline Giza 95 x Ustraly 13 & -1.55 & -2.11 & -1.54 & $-0.52^{\star}$ & 0.09 & 0.09 \\
\hline Giza 95 x Pima S $_{4}$ & $2.13^{*}$ & 5.16 & $2.97^{\star}$ & $0.70^{* *}$ & -0.05 & -0.03 \\
\hline $\begin{array}{ll}\text { LSD } & 0.05\end{array}$ & 2.12 & 5.73 & 2.34 & 0.49 & 0.10 & 0.22 \\
\hline LSD & 2.83 & 7.64 & 3.12 & 0.65 & 0.13 & 0.30 \\
\hline
\end{tabular}

Table 6. Cont.

\begin{tabular}{|l|c|c|c|c|c|}
\hline \multicolumn{1}{|c|}{ Crosses } & LI & UHM & FS & MIC & UI \\
\hline Giza 80 x Karshenky & -0.05 & -0.02 & -0.02 & $0.14^{\star \star}$ & $0.75^{\star \star}$ \\
\hline Giza 80 x Ustraly 13 & 0.10 & $-0.34^{\star \star}$ & 0.11 & $-0.09^{\star}$ & 0.20 \\
\hline Giza 80 x Pima S & -0.04 & $0.36^{\star \star}$ & -0.09 & -0.06 & $-0.95^{\star \star}$ \\
\hline Giza 86 x Karshenky & $-0.20^{\star}$ & $0.22^{\star}$ & 0.06 & $0.25^{\star \star}$ & 0.37 \\
\hline Giza 86 x Ustraly 13 & 0.08 & -0.06 & 0.02 & $-0.14^{\star \star}$ & $-0.68^{\star}$ \\
\hline Giza 86 x Pima S & 0.13 & -0.16 & -0.08 & $-0.11^{\star}$ & 0.31 \\
\hline Giza 90 x Karshenky & 0.12 & 0.02 & -0.01 & -0.05 & -0.50 \\
\hline Giza 90 x Ustraly 13 & -0.14 & 0.14 & -0.08 & $0.16^{\star \star}$ & -0.19 \\
\hline Giza 90 x Pima S & 0.03 & -0.16 & 0.09 & $-0.11^{\star}$ & $0.70^{\star}$ \\
\hline Giza 93 x Karshenky & 0.04 & $0.26^{\star}$ & -0.09 & $-0.15^{\star \star}$ & 0.43 \\
\hline Giza 93 x Ustraly 13 & 0.16 & 0.17 & $0.17^{\star}$ & -0.01 & -0.23 \\
\hline Giza 93 x Pima S & $-0.19^{\star}$ & $-0.43^{\star \star}$ & -0.09 & $0.15^{\star \star}$ & -0.20 \\
\hline Giza 94 x Karshenky & $0.19^{\star}$ & $-0.25^{\star}$ & 0.04 & $-0.24^{\star \star}$ & $-1.09^{\star \star}$ \\
\hline Giza 94 x Ustraly 13 & -0.11 & $0.40^{\star \star}$ & -0.14 & -0.06 & 0.32 \\
\hline
\end{tabular}


Estimation of heterosis and combining ability for yield and fiber quality traits....

\begin{tabular}{|l|c|c|c|c|c|}
\hline Giza 94 x Pima S & -0.08 & -0.14 & 0.10 & $0.30^{\star \star}$ & 0.77 \\
\hline Giza 95 x Karshenky & -0.09 & $-0.23^{\star}$ & 0.02 & 0.03 & 0.04 \\
\hline Giza 95 x Ustraly 13 & -0.08 & $-0.31^{\star \star}$ & -0.08 & $0.14^{\star \star}$ & $0.59^{\star}$ \\
\hline Giza 95 x Pima S & 0.17 & $0.55^{\star \star}$ & 0.06 & $-0.17^{\star \star}$ & $-0.63^{\star}$ \\
\hline LSD 0.05 & 0.19 & 0.22 & 0.17 & 0.09 & 0.56 \\
\hline LSD 0.01 & 0.26 & 0.29 & 0.23 & 0.13 & 0.75 \\
\hline
\end{tabular}

*,** Significant and highly significant at 0.05 and 0.01 probability levels, respectively.

The results of specific combining ability effects for crosses Giza $86 \times$ Ustraly 13, Giza $90 \times$ Pima $S_{4}$, Giza $93 \times$ Karshenky and Giza $95 \times$ Pima $S_{4}$ were significant desirable SCA effects for some yield traits, while, the crosses Giza $90 \times$ Pima $S_{4}$, Giza $93 \times$ Karshenky and Giza $95 \times$ Pima $S_{4}$ were significant desirable SCA effects for some fiber traits. Sorour et al., (2013) found that the best general combiner for most of studied traits was parent (10229 x G. 86). Also the best general combiners for most of studied traits were crosses (10229 x G. 86) x Pima S1, G.45 x G.70, CB.58 x G.70 and CB.58 x G.93. The parent (10229 x G. 86) had the best general combining ability for boll weight, seed cotton yield, lint yield and lint percentage. The crosses CB.58 x G.93 and G.45 x G.70 showed highly significant desirable specific combining ability for boll weight, seed cotton yield, lint yield and number of bolls per plant. Lakho et al., (2016) found that among the parents, NIAB-78, Haridost and CRIS-134 were best general combiners for bolls per plant, boll weight, seed cotton yield per plant and seed index. the cross NIAB- $78 \times$ Chandi- 95 was best specific combiner for bolls per plant and the hybrid Chandi-95×CRIS-134 proved best specific combiner for seed cotton yield per plant, while NIAB$78 \times$ CRIS-134 gave maximum SCA effects for seed index. Swetha et al., (2018) found that among the parents: GSB 40, RHCB 011 and DB 16 were found to be best general combiners for seed cotton yield. Parent TCB 37 and GSB 21 are good combiners for fiber quality traits. Sivia et al., (2020) found that the significant SCA affects were recorded for seed cotton yield from the cross combination AC726 $\times$ H1236, H1476 $x$ H1226, Luxmi PKV X H1226, H1470 X H 1098-I and H1470 X H1236.

\section{Proportional contribution}

Relative percentages of contribution of lines, testers and lines $x$ testers interaction are shown in Table (7). The results showed that lines $x$ tester interaction contribution were higher than tester contribution for all studied traits. However, proportion contribution of lines was higher than of lines $x$ tester interaction contribution and testers for all studied traits. Al-Hibbiny (2011) found that proportion contribution of lines $x$ tester interaction was higher than of lines and testers for all studied characters, except lint percentage. Lines contribution was higher than testers contribution for most studied traits. Chapara et al., (2020) found that the line $x$ tester interactions made greater contribution to the total variance for most of the traits i.e. boll number per plant, boll weight, lint index, lint yield, micronaire.

\section{Genetic parameters}

Knowledge of gene action helps in the selection of parents for using in the hybridization programs and also in the choice of appropriate breeding procedure for the genetic improvement of various quantitative characters. Hence, insight into the nature of gene action involved in the expression of various quantitative characters is essential to a plant breeder for starting a judicious breeding program. The genetic variance component and dominance degree ratio were calculated for all studied traits are 
presented in Table (8). The results indicated that the non-additive of genetic parameters were larger than additive genetic variance with respect to all studied traits except lint percentage, seed index, lint index and upper half mean.

These results indicated that nonadditive effects play a major role in the expression of these traits, while additive effects had a minor role. This indicated that the hybridization program would be effective in improvement of most studied traits. The importance of non-additive genetic variances was verified by the average degree of dominance which is more than one for most traits. This indicated that the overdominance played an important role of the dominance component. Basal et al., (2009) cleared that the predominance of non-additive gene action was found for all traits, except for the upper half mean (UHM) and fiber strength, which were controlled by an additive type gene action due to the high GCA variance. Chapara et al., (2020) found that the ratio of $\sigma 2 \mathrm{GCA} / \sigma 2 \mathrm{SCA}$ was smaller than zero for all the characters indicating predominance of non-additive gene action (dominant or epistasis) in the inheritance of investigated traits except lint index. Nand et al., (2020) found that the magnitude of GCA variances was higher than SCA variance suggesting per-ponderance of additive gene effects for almost all the traits.

Table 7. Proportional contributions of lines, testers and their interaction for yield, yield components and fiber traits.

\begin{tabular}{|c|c|c|c|}
\hline Traits & Lines & Testers & $\begin{array}{c}\text { Lines } \mathbf{x} \\
\text { Testers }\end{array}$ \\
\hline No. of bolls/plant & 65.54 & 10.08 & 24.38 \\
\hline Seed cotton yield/plant & 77.90 & 5.48 & 16.62 \\
\hline Lint cotton yield/plant & 73.29 & 7.55 & 19.15 \\
\hline Lint percentage & 90.64 & 2.03 & 7.33 \\
\hline Boll weight & 75.15 & 0.99 & 23.86 \\
\hline Seed index & 95.83 & 0.29 & 3.88 \\
\hline Lint index & 93.43 & 0.59 & 5.98 \\
\hline Upper half mean & 94.63 & 1.04 & 4.33 \\
\hline Fiber strength & 82.52 & 5.28 & 12.20 \\
\hline micronaire reading & 65.00 & 8.57 & 26.43 \\
\hline Uniformity index & 68.28 & 6.70 & 25.02 \\
\hline
\end{tabular}

Table 8. The partitioning of the genetic variance for yield, yield components and fiber traits.

\begin{tabular}{|c|c|c|c|c|c|c|c|c|c|c|c|}
\hline $\begin{array}{c}\text { Genetic } \\
\text { parameters } \\
\text { And } \\
\text { heritability }\end{array}$ & NB/P & SCY/P & LCY/P & L\% & BW & SI & LI & UHM & FS & MIC & UI \\
\hline GCA & 0.91 & 16.53 & 2.47 & 0.15 & 0.001 & 0.04 & 0.02 & 0.15 & 0.004 & 0.005 & 0.07 \\
\hline SCA & 6.04 & 64.20 & 11.88 & 0.18 & 0.01 & 0.02 & 0.02 & 0.12 & 0.01 & 0.04 & 0.51 \\
\hline
\end{tabular}


Estimation of heterosis and combining ability for yield and fiber quality traits....

\begin{tabular}{|c|c|c|c|c|c|c|c|c|c|c|c|}
\hline$\sigma^{2} \mathrm{~A}$ & 1.82 & 33.06 & 4.94 & 0.30 & 0.002 & 0.08 & 0.04 & 0.30 & 0.01 & 0.01 & 0.14 \\
\hline$\sigma^{2} \mathrm{D}$ & 6.04 & 64.20 & 11.88 & 0.18 & 0.01 & 0.02 & 0.02 & 0.12 & 0.01 & 0.04 & 0.51 \\
\hline$\left(\sigma^{2} \mathrm{D} / \sigma^{2} \mathrm{~A}\right)^{1 / 2}$ & 1.82 & 1.39 & 1.55 & 0.77 & 2.24 & 0.50 & 0.71 & 0.63 & 1.00 & 2.00 & 1.91 \\
\hline$\sigma^{2} \mathrm{G}$ & 6.95 & 80.73 & 14.35 & 0.33 & 0.01 & 0.06 & 0.04 & 0.28 & 0.01 & 0.04 & 0.58 \\
\hline$\sigma^{2} \mathrm{E}$ & 3.36 & 24.46 & 4.09 & 0.18 & 0.01 & 0.04 & 0.03 & 0.04 & 0.02 & 0.01 & 0.24 \\
\hline$\sigma^{2} \mathrm{Ph}$ & 10.31 & 105.19 & 18.44 & 0.51 & 0.01 & 0.10 & 0.07 & 0.31 & 0.03 & 0.05 & 0.82 \\
\hline $\mathrm{H}_{\mathrm{b}}^{2}$ & 67.41 & 76.74 & 77.83 & 65.19 & 53.53 & 61.69 & 58.30 & 88.47 & 32.24 & 86.72 & 70.98 \\
\hline $\mathrm{H}_{\mathrm{n}}^{2}$ & 8.82 & 15.71 & 13.38 & 29.73 & 8.04 & 41.70 & 31.99 & 49.03 & 14.03 & 9.05 & 8.74 \\
\hline
\end{tabular}

\section{Heritability}

The results of heritability in broad and narrow senses are illustrated in Table (8). The results revealed that broad sense heritability $\left(\mathrm{h}_{\mathrm{b}}^{2} \%\right)$ estimates were larger than the corresponding values of narrow sense heritability $\left(h^{2} \%\right)$ for all studied traits. The highest broad sense heritability estimates was observed in case of UHM with values of $88.47 \%$ and the lowest was for fiber strength with value of $32.24 \%$, while for narrow sense heritability, it was ranged from $8.04 \%$ to $49.03 \%$ for boll weight and upper half mean, respectively. Sorour et al., (2013) found that heritability estimates in narrow sense were low to high for all the studied traits, ranged from $32.17 \%$ for seed cotton yield to $91 \%$ for boll weight. AL-Hibbiny (2015) found that high heritability estimates in broad-sense $(>50 \%)$ were detected for all traits studied at the two crosses, except seed cotton yield/plant of the cross II and fiber fineness of the cross I. Heritability estimates in narrow-sense ranged from 0.00 to $37.51 \%$ for boll weight of the cross I and $2.5 \%$ span length of the cross II, respectively.

\section{REFERENCES}

Ahuja S. and S. Dhayal (2007). Combining ability estimates for yield and fibre quality traits in $4 \times 13$ line $\times$ tester crosses of Gossypium hirsutum. L.; Euphytica 153:87-98.
AL-Ameer, M. A. (2015). Estimation of heterosis and gene action using Line xTester analysis for yield components and fiber characters in Gossypium barbadense, L. J. Agric. Res., Kafr ElSheikh Univ. 41 (2): 546 - 564.

Al-Hibbiny, Y.I.M. (2015) Estimation of heterosis, combining ability and gene action by using line $X$ tester analysis in cotton (Gossypium barbadense L.). Egypt. J. Plant Breed. 19(2):385 - 405.

AL-Hibbiny, Y.I.M., A.H. Mabrouk and B.M. Ramadan (2020). Generation means analysis for some quantitative characters in cotton. Menoufia J. Plant Prod., Vol. 5 June (2020): 111 - 123.

Al-Hibbiny, Y.I.M. (2011). Breeding of some boll characters and its contents in cotton. Ph.D. Thesis, Agron. Dept. Fac. Agric., Tanta Univ. Egypt.

Allard, R.W. (1960). Principles of Plant Breeding. John Wiley, New York .

Balcha M., Mohammed W. and $Z$. Desalegn (2019) Combining ability and heritability for yield, yield related and fiber quality traits in cotton (Gossypium spp.) at Werer, Ethiopia. Inter. J. of Plant Breeding and Genetics. 6 (8), 1-14.

Baloch, M. J., J. A. Solangi, W. A. Jatoi, I. H. Rind and F. M. Halo (2014). Heterosis and specific combining ability esti.mates for assessing potential crosses to develop $F_{1}$ hybrids in upland cotton. Pak. J. Agri., Agril. Engg., Vet. Sci., 30 (1): 8-18. 
Basal, H., A. Unay, O. Canavar and I. Yavas (2009). Combining ability for fiber quality parameters and withinboll yield components in intraspecific and interspecific cotton populations Spanish Agric. Res. 7(2), 364-374.

Basbag, S., R. Ekinci and O. Gencer (2007). Combining ability and heterosis for earliness characters in line $\times$ tester population of Gossypium hirsutum L. Hereditas.144:185-190.

Batool, S., N.U. Khan, K. Makhdoom, Z. Bibi, G. Hassan and K.B. Marwat (2010). Heritability and genetic potential of upland cotton genotypes for morpho-yield traits. Pakistan Journal of Botany. 42(2):1057-1064.

Chapara, R., Rani S. M. and Y. Satish (2020). Combining ability studies in cotton (Gossypium hirsutum L.) for yield and fibre quality parameters. International Journal of Chemical Studies 2020; 8(2): 523-527.

Cochran, W.C. and G.M. Cox (1957). Experimental Design. $2^{\text {nd }}$ ed., John Wiley and Sons Inc., New York. U.S.A.

Kempthorne, O. (1957). An Introduction to Genetic Statistics. Iowa State Univ. John Wiley and Sons Inc. New York, U.S.A.

Khan, S.A. (2010). Combining ability analysis in Upland Cotton (G. hirsutum). M.Sc (Hons.) Thesis, Khyber Pakhtunkhwa Agril. Univ. Peshawar, Pakistan.

Konak, C., A. Unay, E. Serter and H. Basal (1999). Estimation of combining ability effects, heterosis and heterobeltiosis by line $x$ tester method in maize. Turkish J Field Crops. 4:1-9.

Lakho, R. H., A. A. Soomro, M. A. R. Rashid and S. Memon (2016). Determination of general and specific combining ability of five Upland Cotton cultivars. Journal of Agricultural Science; 8,. 3. 106-111.

Lingaraja, L., R.S. Sangwan, S. Nimbal, Omender Sangwan and Sukhdeep Singh (2017). Heterosis Studies For
Economic and Fibre Quality Traits in Line $X$ Tester Crosses of Upland Cotton (Gossypium hirsutum L.). Int. J. Pure App. Biosci. 5 (2): 240-248 (2017).

Mabrouk, A.H., M.A.A. El-Dahan and Eman M.R. Saleh (2018). Diallel analysis for yield and fiber traits in cotton. Egypt. J. Plant Breed. 22(1):109- 124 (2018).

Mahrous, H. (2018). Line $\times$ Tester analysis for yield and fiber quality traits in Egyptian Cotton under heat conditions. J. Plant Production, Mansoura Univ., 9 (6): 573 - 578.

Mather, K. (1949). Biometrical Genetics. Dover Publication. Inc. New York.

Mert, M., O. Gencer, Y. Akiscan and K.Boyaci (2003). Determination of superior parents and hybrid combination in respect to lint yield and yield components in cotton (Gossypium hirsutum L.). Turk J. Agric. For. 27:337-343.

Nand, K.K., V.N. Chinchane, G.R. Gopal and S.G. Puri (2020). Studies on combining ability for yield, yield contributing and fibre quality traits in Desi Cotton (Gossypium arboreum L.). Journal of Pharmacognosy and Phytochemistry 9(2): 12-14.

Samreen, K., M.J. Baloch, Z.A. Soomro, M.B. Kumbhar, N.U. Khan, N. Kumboh, W.A. Jatoi and N.F. Veesar (2008). Estimating combining ability through Line $x$ Tester analysis in Upland Cotton. Sarhad J. Agric.24, 4, 581-586.

Singh, R.K. and B.D. Chaudhary (1979). Biometrical Methods in Quantitative Genetic Analysis. $2^{\text {nd }}$ ed., Kalyani, Publishers, Daryagnai, New Delhi.

Sivia, S. S., S. S. Siwach, O. Sangwan, L. Lingaraja. and R. D. Vekariya (2020). Combining Ability Estimates for Yield Traits in Line $x$ Tester Crosses of Upland Cotton (Gossypium hirsutum). Int. J. Pure App. Biosci. 5 (1): 464-474.

Sorour, F.A., M. S. Abdel- Aty, W.M.B. Yehia and H.M.K. Kotb (2013). 
Heterosis and combining ability in some cotton crosses in two different environments: 1- Yield and yield components traits. J. Plant Production, Mansoura Univ., 4 (11): 1707 - 1723.

Sprague, G.F. and L.A. Tatum (1942). General vs. specific combining ability in single crosses of corn. J Am. Soc. Agron. 34:923-932.

Steel, R.G.D. and J.H. Torrie (1985). Principles and Procedures of Statistics. Mc Graw-Hill, Book company, Inc., New York.
Swetha, S, J.M. Nidagundi, J.R. Diwan, R. Lokesha, A.C. Hosmani and A. Hadimani (2018). Combining ability studies in cotton (Gossypium barbadense L.). Journal of Pharmacognosy and Phytochemistry 7(1): 638-642.

Yehia, W.M.B. and E. F. EL-Hashash (2019). Combining Ability Effects and Heterosis Estimates through Line $x$ Tester Analysis for Yield, Yield Components and Fiber Traits in Egyptian Cotton. Elixir Agriculture (3): 53238-53246.

تقدير قوة الهجين والقدرة علي التآلف لصفات المحصول وجودة الالياف باستخدام تحليل السلالة x الكشاف في أقطان الباربادنس

$$
\text { هبه حسين السيد حامد، صلاح الدين رشاد نصر سعيد }
$$

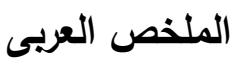

أجريت هذه الاراسة في محطة البحوث الزراعية بسدس - معهل بحوث القطن - مركز البحوث الزراعية - مصر خلال

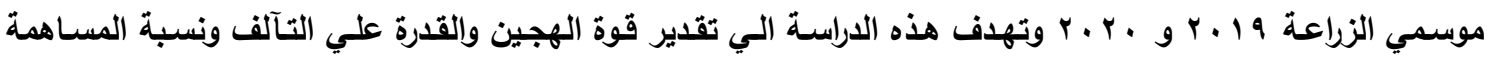

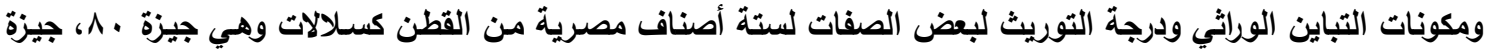

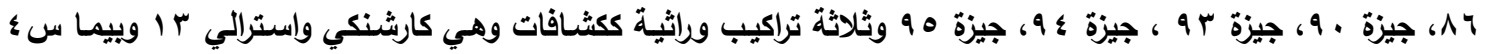

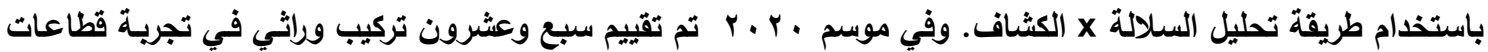

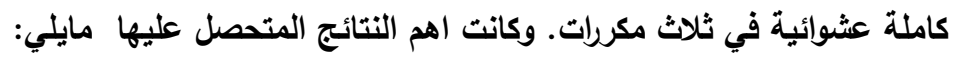

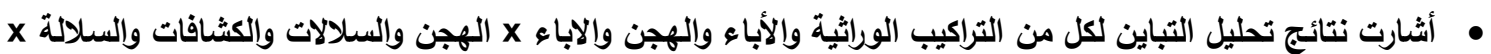

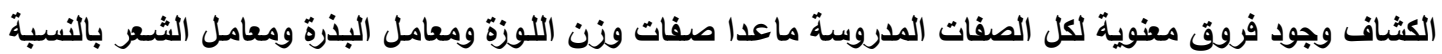
للكثافات وصفة متانة التيلة بالنسبة للسلالة x الكثاف.

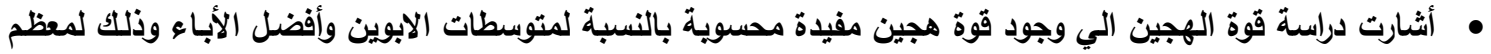

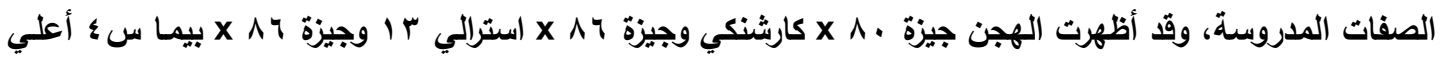

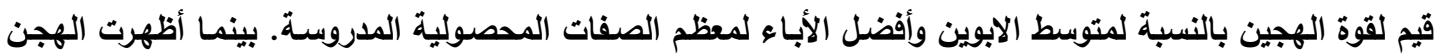

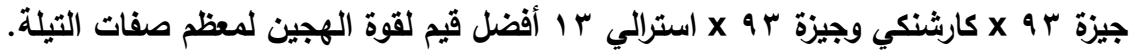

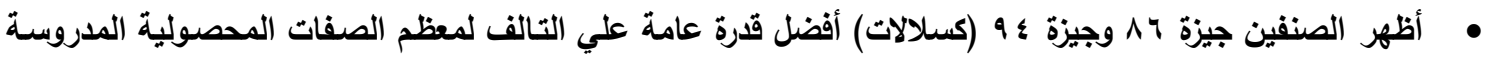
بينما أظهر الصنف جيزة به (كسلالة) أفضل قدرة عامة علي التالف لكل صفات التيلة المدروسة. كما أظهرت الهجن

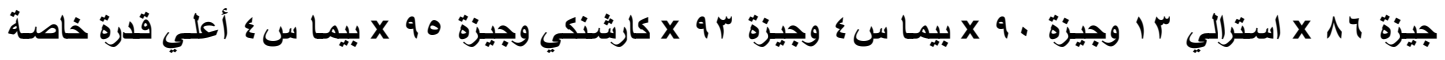

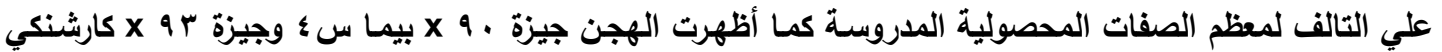
وجيزة ه 9 ه بيما س ؛ أعلي قدرة خاصة علي التالف لمعظم صفات التيلة المدروسة. 
• أظهر تقدير نسبة المسـاهمة الـي أن مسـاهمة السـلالات أعلـي مـن مسـاهمة كل مـن تفاعل السـلاله x الكثـاف والكثافات لكل الصفات المدروسة.

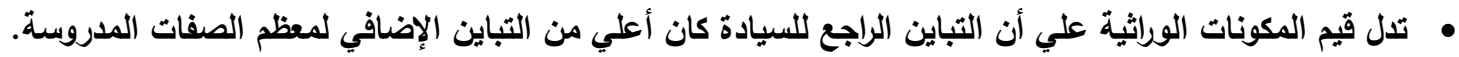

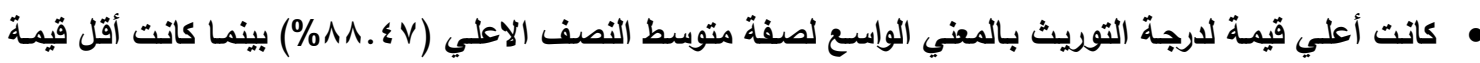

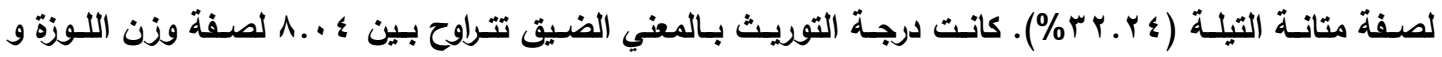

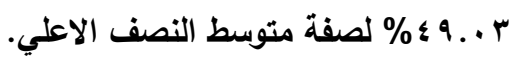
عموما فانه يمكن التوصيه باستخدام الصنفين جيزة 1 و وجيزة ؛ 9 في برامج التربية لتحسين وزيادة القدرة الانتاجية

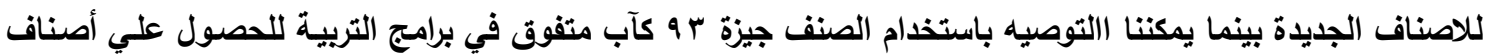
جليدة عالية الجودة.

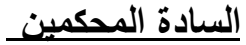
أ.د/ عيسى محمود غنيـم مركز البحوث الزراعية - الجيزة

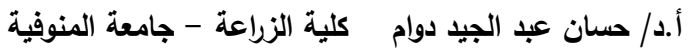

